\title{
Detection of Diastolic Abnormality by Dyssynchrony Imaging Correlation With Coronary Artery Disease in Patients Presenting With Visibly Normal Wall Motion
}

\author{
Toshinari Onishi, MD; Masaaki Uematsu, MD; Shinsuke Nanto, MD; \\ Takakazu Morozumi, MD; Tetsuya Watanabe, MD; Masaki Awata, MD; \\ Osamu Iida, MD; Fusako Sera, MD; Seiki Nagata, MD
}

\begin{abstract}
Background Post-systolic shortening (PSS) is a sensitive indicator of myocardial ischemia.
Methods and Results We have developed a tissue Doppler imaging technique that portrays PSS, and whether PSS correlates with coronary artery disease (CAD) was investigated in 186 patients presenting with chest pain and normal echocardiograms. Delays of the displacement peaks from end-systole were calculated in the apical views and displayed from green $(0 \mathrm{~ms})$ to red $(\geq 100 \mathrm{~ms})$ : detection of diastolic abnormality by dyssynchrony imaging (DADI). CAD was judged positive by DADI when the left ventricular segments were color-coded red. Patients subsequently underwent thallium-201 myocardial perfusion single-photon emission computed tomography $(n=150)$, coronary angiography $(\mathrm{CAG}, \mathrm{n}=74)$, or both $(\mathrm{n}=37)$. $\mathrm{CAD}(-)$ was defined as negative scintigraphy test and/or no significant coronary artery stenosis by CAG. In 43 patients $(23 \%), \mathrm{CAD}(+)$ was confirmed by CAG as $>75 \%$ diameter stenosis. DADI predicted CAD with sensitivity of $60 \%$, specificity of $75 \%$, predictive accuracy of $72 \%$, positive predictive value of $42 \%$, and negative predictive value of $86 \%$. Among 74 patients who underwent CAG, sensitivity was best for the left anterior descending artery.

Conclusions DADI detected the regional diastolic abnormality, which correlated with the presence of CAD in patients presenting with visibly normal wall motion. (Circ J 2009; 73: 125-131)
\end{abstract}

Key Words: Coronary artery disease; Echocardiography; Tissue Doppler imaging

C hest pain still remains a challenge for clinicians. The possibility of coronary artery disease (CAD) cannot be excluded by normal electrocardiograms or normal echocardiograms at rest. Exercise electrocardiography has found wide acceptance for CAD identification because of its low cost. However, the sensitivity and specificity range from $65 \%$ to $80 \%$, and a substantial number of patients are unable to exercise because of comorbidities. Dobutamine stress echocardiography may be a solution, but its interpretation is largely operator dependent? It would be desirable if the detection of CAD could be done objectively without any provocation tests.

\section{Editorial p37}

Post-systolic shortening (PSS) is a delayed ejection motion of the myocardium occurring after aortic valve closure during the generally prolonged isovolumic relaxation time. Both clinical $^{3-5}$ and animal studies ${ }^{6-10}$ have demonstrated that PSS is related to myocardial ischemia.

(Received July 28, 2008; revised manuscript received August 25, 2008; released online November 13, 2008)

Cardiovascular Division, Kansai Rosai Hospital, Amagasaki, Japan This work was partly supported by research funds to promote hospital functions of the Japan Labour Health and Welfare Organization, Kawasaki, Japan.

Mailing address: Masaaki Uematsu, MD, Cardiovascular Division, Kansai Rosai Hospital, 3-1-69 Inabaso, Amagasaki 660-8511, Japan. E-mail: uematsu@kanrou.net

All rights are reserved to the Japanese Circulation Society. For permissions, please e-mail: cj@j-circ.or.jp
We have previously demonstrated that the presence of a positive myocardial velocity during the isovolumic relaxation phase (VIR) detected by spectral tissue Doppler imaging (TDI), which corresponds to PSS, correlates with the presence of CAD among patients presenting with visibly normal left ventricular (LV) contraction!1

Color-coded TDI has facilitated quantitative assessment of ventricular wall motion, ${ }^{12-18}$ so it would be helpful if the presence of PSS was made readily visible by this technique without the need for mapping. In the present study, we aimed to develop a color-coded TDI technique that detects PSS on 2-dimensional (D) echocardiograms: detection of diastolic abnormality by dyssynchrony imaging (DADI). We also prospectively investigated the correlation of the diastolic abnormality detected by DADI with the presence of $\mathrm{CAD}$ among patients complaining of chest pain and presenting with visibly normal LV wall motion.

\section{Methods}

\section{Patients}

Between November 2005 and September 2006, we enrolled 195 consecutive patients complaining of chest pain and presenting with normal LV wall motion evaluated by standard echocardiography in the cardiology outpatient clinic. Consent was given to undergo thallium-201 myocardial perfusion single-photon emission computed tomography (SPECT) and/or coronary angiography (CAG), in addition to echocardiography including TDI. Patients presenting with abnormal echocardiograms, such as wall motion abnormalities, significant valvular disease, dilated or restrictive 
cardiomyopathies, LV hypertrophy (the interventricular septum or the posterior wall thickness $\geq 12 \mathrm{~mm}$ ), pulmonary hypertension (tricuspid valve regurgitation velocity $\geq 2.5 \mathrm{~m} / \mathrm{s}$ ), including a history of prior myocardial infarction, previous coronary angioplasty or bypass grafting, atrial fibrillation or flutter, pacemaker implantation, left bundle branch block, abnormal echocardiograms, and congestive heart failure, were excluded. Nine patients were further excluded from the analysis because of inadequate ultrasound images.

The remaining 186 patients ( 88 women, mean age 63 years, age range 17-90 years) underwent spectral and colorcoded TDI at rest, in addition to standard echocardiography (Fig 1). Subsequently, patients underwent thallium-201

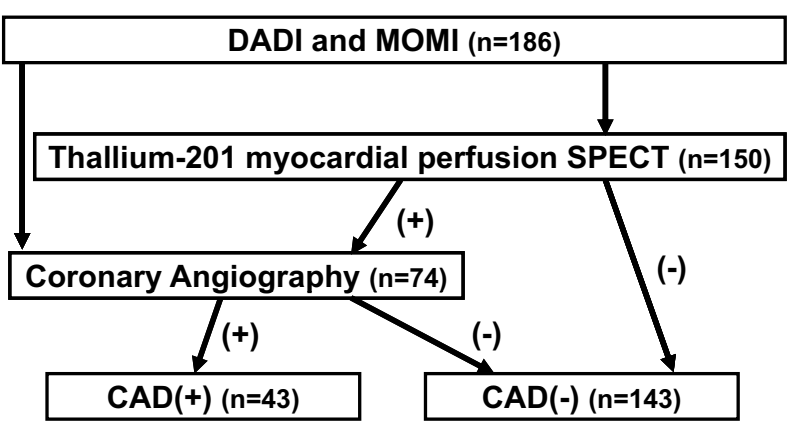

Fig 1. Block diagram of the study protocol. MOMI, mapping of myocardial velocity imaging; DADI, diastolic abnormality by dyssynchrony imaging; SPECT, single-photon emission computed tomography; CAD, coronary artery disease. myocardial perfusion SPECT $(n=150)$ and/or CAG $(n=74)$. The study protocol was approved by the Ethics Committee of Kansai Rosai Hospital. All patients gave written informed consent.

\section{Echocardiography and TDI}

All patients underwent routine echocardiography, spectral TDI, and color-coded TDI using a cardiac ultrasound diagnostic apparatus (Aplio SSA-770A, Toshiba, Tokyo, Japan) with a 3.6-MHz sector transducer. Echocardiograms were obtained in the left lateral position at end expiration. No stress was placed on the subjects. LV end-diastolic and end-systolic dimensions were measured on 2-D echocardiography-guided M-mode echocardiograms. ${ }^{19}$ TDI was performed in the apical 4- and 2 chamber views.

\section{Detection of Diastolic Abnormality by Dyssynchrony Imaging (DADI)}

Displacement was calculated from the color-coded TDIderived velocity data using online software (TDI-Q, Toshiba) ${ }^{20,21}$ In brief, myocardial displacement along the ultrasound beam direction was calculated by temporal integration of the velocity at each myocardial point on the beam. Importantly, this software is capable of Doppler tissue tracking along the beam direction, thereby enabling accurate measurement of the displacement. The accuracy of displacement measured with this system was validated by an in vitro study?1 End-systole was estimated from the Doppler velocity when the sum of the velocities obtained from the entire image reached the velocity closest to zero. Subsequently, a time window was set from end-systole in such a way that when the peak of the displacement occurred at the
A

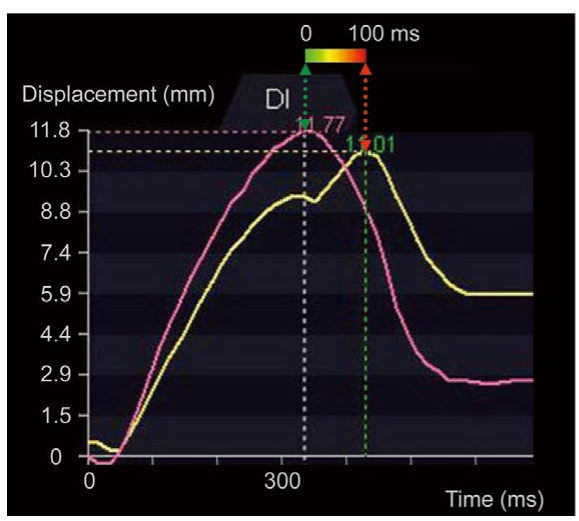

B

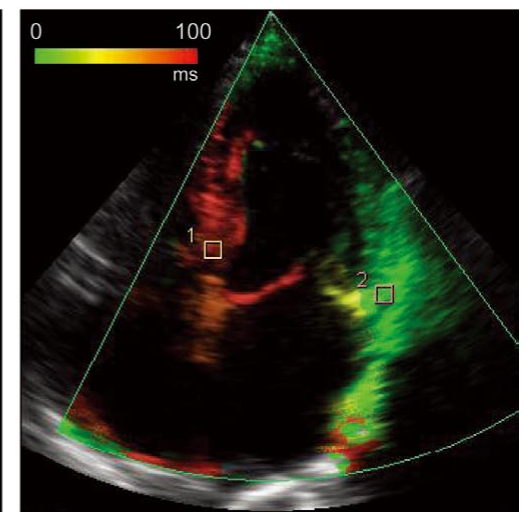

Fig 2. Principles of detection of diastolic abnormality by dyssynchrony imaging (DADI). (A) Displacement curves calculated from the velocity measured from the interventricular septum (yellow line) and from the lateral wall (pink line) in a patient with coronary artery disease. (B) The peak of the pink displacement curve is seen at the time of end-systole, whereas the peak of the yellow curve is delayed from end-systole. DADI portrays the regional delay in the peak of displacement on 2-dimensional echocardiograms by detecting the difference in timing within a specified time interval from end-systole. The color coding ranges from green (earliest) to red (latest) within the specified interval.

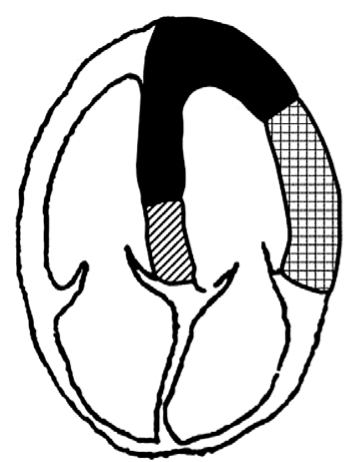

4-chamber
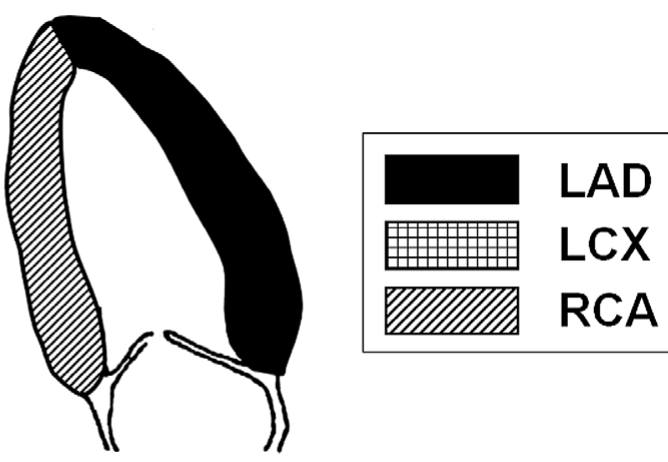

2-chamber
Fig 3. Regional wall segments per coronary artery perfusion used for the detection of coronary artery disease (CAD) by detection of diastolic abnormality by dyssynchrony imaging (DADI). CAD was judged positive by DADI when part of the left ventricle was color-coded red (regional delay in the displacement peak $\geq 100 \mathrm{~ms}$ ) in accordance with the anatomy of the coronary artery branches. LAD, left anterior descending artery; LCX, left circumflex artery; RCA, right coronary artery. 
Table 1 Characteristics of Patients With and Without CAD

\begin{tabular}{|c|c|c|c|}
\hline & $C A D(+)(n=43)$ & $C A D(-)(n=143)$ & $P$ value \\
\hline Female & $12(28 \%)$ & $76(53 \%)$ & $<0.01$ \\
\hline Age (years) & $65 \pm 9$ & $63 \pm 12$ & $N S$ \\
\hline \multicolumn{4}{|l|}{ Coronary risk factors } \\
\hline Hypertension & $36(84 \%)$ & $83(58 \%)$ & $<0.01$ \\
\hline Dyslipidemia & $33(77 \%)$ & $62(43 \%)$ & $<0.01$ \\
\hline Hyperuricemia & $8(19 \%)$ & $24(17 \%)$ & $N S$ \\
\hline Diabetes mellitus & $17(40 \%)$ & $33(23 \%)$ & $<0.05$ \\
\hline Current smoking & $16(37 \%)$ & $31(22 \%)$ & $<0.05$ \\
\hline$S B P(m m H g)$ & $138 \pm 18$ & $138 \pm 16$ & $N S$ \\
\hline$D B P(m m H g)$ & $75 \pm 12$ & $77 \pm 10$ & $N S$ \\
\hline Heart rate (beats/min) & $65 \pm 9$ & $68 \pm 12$ & $N S$ \\
\hline \multicolumn{4}{|l|}{ Echocardiographic data } \\
\hline $\operatorname{LVDd}(\mathrm{mm})$ & $48 \pm 4$ & $45 \pm 4$ & $<0.01$ \\
\hline LVDs $(\mathrm{mm})$ & $29 \pm 3$ & $28 \pm 4$ & $N S$ \\
\hline$F S(\%)$ & $40 \pm 4$ & $40 \pm 5$ & $N S$ \\
\hline IVSth $(\mathrm{mm})$ & $10 \pm 1$ & $9 \pm 1$ & $<0.01$ \\
\hline LVPWth (mm) & $10 \pm 1$ & $9 \pm 1$ & $<0.01$ \\
\hline
\end{tabular}

$C A D$, coronary artery disease; $N S$, not significant; $S B P$, systolic blood pressure; DBP, diastolic blood pressure; $L V D d$, left ventricular diameter at end-diastole; LVDs, left ventricular diameter at end-systole; FS, fractional shortening of the left ventricular shortaxis diameter; IVSth, wall thickness of the interventricular septum; LVPWth, wall thickness of the left ventricular posterior wall.

timing of the estimated end-systole, it was color-coded green, and that when the peak of the displacement was delayed from the estimated end-systole by more than $100 \mathrm{~ms}$, it was color-coded red. In this way, DADI color-coded a delay in the peak of displacement occurring in the whole $\mathrm{LV}$ and displayed the anatomical distribution of the delay as a color-coded parametric image on the 2-D echocardiogram (Fig 2). Thus, DADI portrayed regional PSS on 2-D echocardiograms. CAD was judged positive by DADI when part of the LV was color-coded red (regional delay in the displacement peak $\geq 100 \mathrm{~ms}$ ) in accordance with the anatomy of the coronary artery branches (Fig 3 ). The initial time window, which started from the estimated end-systole, was set to $100 \mathrm{~ms}$ based on our previous data using spectral TDI! ${ }^{1}$ Effects of the time window were evaluated in this study at 50,100 , and $150 \mathrm{~ms}$.

Intraobserver agreement of DADI was assessed by a single investigator (T.O.) in a randomly selected 50 patients on 2 separate occasions. Interobserver agreement was also assessed in the same patient population by 2 independent observers (T.O. and S.O.).

\section{Mapping of Myocardial Velocity Imaging (MOMI)}

Spectral TDI measurements were also done at the annular and mid-LV levels in the apical 4- and 2-chamber views (8 points in each patient) as previously described ${ }^{10}$ Positive VIR was defined as an upward spike between the end of the systolic ejection phase and the onset of the early diastolic relaxation phase that lasted longer than $100 \mathrm{~ms}$. We determined whether positive VIR was present on the spectral TDI tracings. CAD was considered present if positive VIR was present in 1 or more of the 8 points in each subject.

\section{Thallium-201 Myocardial Perfusion SPECT}

Within 1 week of TDI, 150 patients $(81 \%)$ underwent thallium-201 SPECT with bicycle exercise testing. A dose of $3.0 \mathrm{mCi}$ of thallium-201 was injected intravenously at near-peak exercise, and the exercise was continued for another minute. The patients were scanned with a dualdetector SPECT system equipped with a low-energy, parallel-hole, all-purpose collimator (Optima, GE Medical System, Wakeshaw, WI, USA). The thallium-201 stress images were obtained within $6 \mathrm{~min}$ of thallium injection. Redistribution imaging was performed $4 \mathrm{~h}$ later using the same acquisition measurements?2 Identification of the redistribution phenomenon was judged as positive for CAD by an independent physician who was unaware of the results of MOMI and DADI.

$C A G$

Within 3 weeks of TDI, 74 patients $(40 \%)$ underwent CAG with multiple views. CAG was interpreted by consensus opinion of 2 physicians who were unaware of the results of SPECT, MOMI or DADI. Coronary narrowing in 1 or more major vessels was graded by visual analysis according to the lumen diameter involved: $\leq 25 \%, 26-50 \%$, $51-75 \%, 76-90 \%, 91-99 \%$, or $100 \%$. Critical coronary artery stenosis was defined as $>75 \%$ diameter stenosis. CAD was diagnosed when patients had a critical coronary artery stenosis on CAG. Hence, in this study the presence of CAD was confirmed in all patients by CAG (Fig 1).

\section{Statistical Analysis}

Numerical variables are expressed as mean \pm SD. Unpaired t-test was used for the comparison of patients with and without CAD. We considered results were significant when $\mathrm{P}<0.05$. The optimal cut-off values of the duration of positive VIR and of the time window from end-systole in DADI for discriminating normal from patients with CAD were determined by receiver-operating characteristic curves.

\section{Results}

\section{Patient Characteristics}

CAD was finally diagnosed by CAG in 43 patients (23\%) among the 186 enrolled in this prospective study; 113 patients $(61 \%)$ were diagnosed as normal by thallium-201 myocardial perfusion SPECT and $30(16 \%)$ by CAG. No significant differences were found between the patients with and without CAD regarding age, hyperuricemia, blood pressure, heart rate, LV diameter at end-systole, and fractional shortening. The proportion of men, hypertension, dyslipidemia, diabetes mellitus, current smoking, LV diameter at end-diastole, wall thickness of the interventricular 

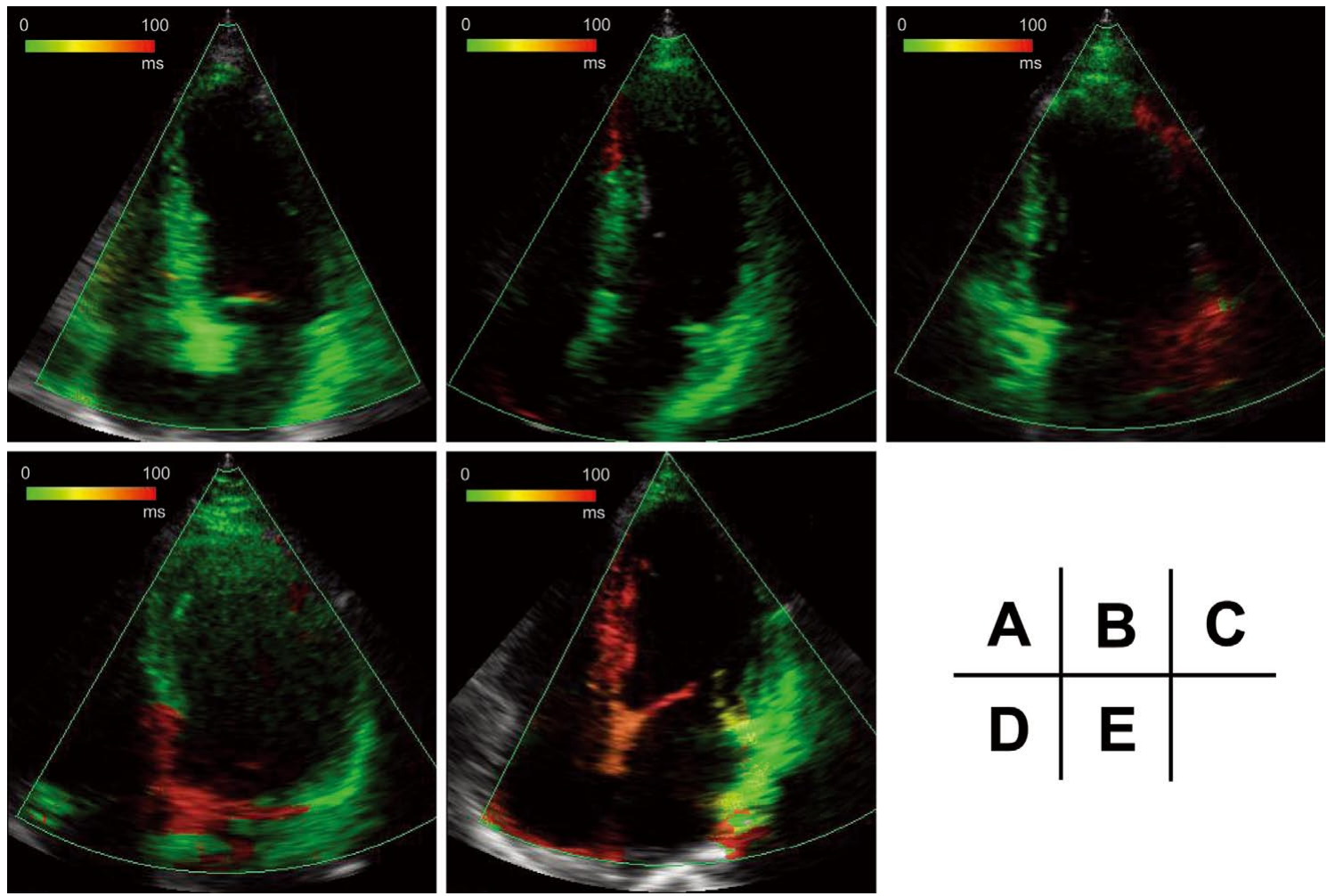

Fig 4. Representative images of detection of diastolic abnormality by dyssynchrony imaging (DADI) in the 4-chamber view obtained from a normal subject $(\mathbf{A})$, patient with a 1-vessel disease of the left anterior descending artery (LAD) (B), 1-vessel disease of the left circumflex artery (LCX) (C), 1-vessel disease of the right coronary artery (RCA) (D), and a patient with 2-vessel disease of the LAD and RCA (E). (A) In the normal subject the entire left ventricle is displayed green by DADI. (B) In contrast, the apical septum is color-coded red when the LAD is diseased. (C) The lateral wall, which is supplied by LCX, is color-coded red when the LCX has a 90\% diameter stenosis. (D) Only the basal septum is color-coded red when the RCA is diseased. (E) The entire interventricular septum is color-coded red from the apex to the base in 2-vessel disease of the LCX and RCA.

Table 2 Correlations of MOMI and DADI With CAD

\begin{tabular}{cccccc}
\hline \hline Cut-off levels & Sensitivity, $\%$ & Specificity, $\%$ & Accuracy, $\%$ & PPV, $\%$ & NPV, $\%$ \\
\hline MOMI & & & & & \\
50 & 85 & 33 & 45 & 27 & 88 \\
100 & 66 & 54 & 56 & 30 & 84 \\
150 & 7 & 97 & 77 & 43 & 78 \\
DADI & 77 & 46 & 53 & 30 & 87 \\
50 & 60 & 75 & 72 & 42 & 86 \\
100 & 28 & 89 & 75 & 43 & 80 \\
150 & &
\end{tabular}

MOMI, mapping of myocardial velocity imaging; DADI, detection of diastolic abnormality by dyssynchrony imaging; PPV, positive predictive value; $N P V$, negative predictive value; $C A D$, coronary artery disease.

septum or LV posterior wall were greater in patients with CAD than in those without CAD (Table 1).

\section{Detection of CAD by DADI}

Fig 4 shows representative DADI images from a normal subject and from patients with a variety of CAD: 1-vessel disease involving the left anterior descending artery (LAD), 1-vessel disease of the left circumflex artery (LCX), 1-vessel disease of the right coronary artery (RCA), and 2-vessel disease involving the RCA and LAD. The distribution of PSS, which reflected the presence of coronary artery stenosis in accordance with the coronary perfusion area, was successfully demonstrated by DADI in these images.

\section{Comparison Between MOMI and DADI}

The overall sensitivity, specificity, predictive accuracy, positive predictive value, and negative predictive value for detecting CAD by MOMI and DADI are shown in Table 2. Receiver-operating characteristic analysis indicated that the duration of positive VIR of $100 \mathrm{~ms}$ was the best cut-off value for MOMI to discriminate normal from CAD; the time window of $100 \mathrm{~ms}$ was the best cut-off value for DADI. DADI predicted the presence of CAD with sensitivity of $60 \%$, specificity of $75 \%$, predictive accuracy of $72 \%$, positive predictive value of $42 \%$, and negative predictive value of $86 \%$ when a time window of $100 \mathrm{~ms}$ was used as the cutoff value. MOMI yielded sensitivity of $66 \%$, specificity of $54 \%$, predictive accuracy of $56 \%$, positive predictive value 

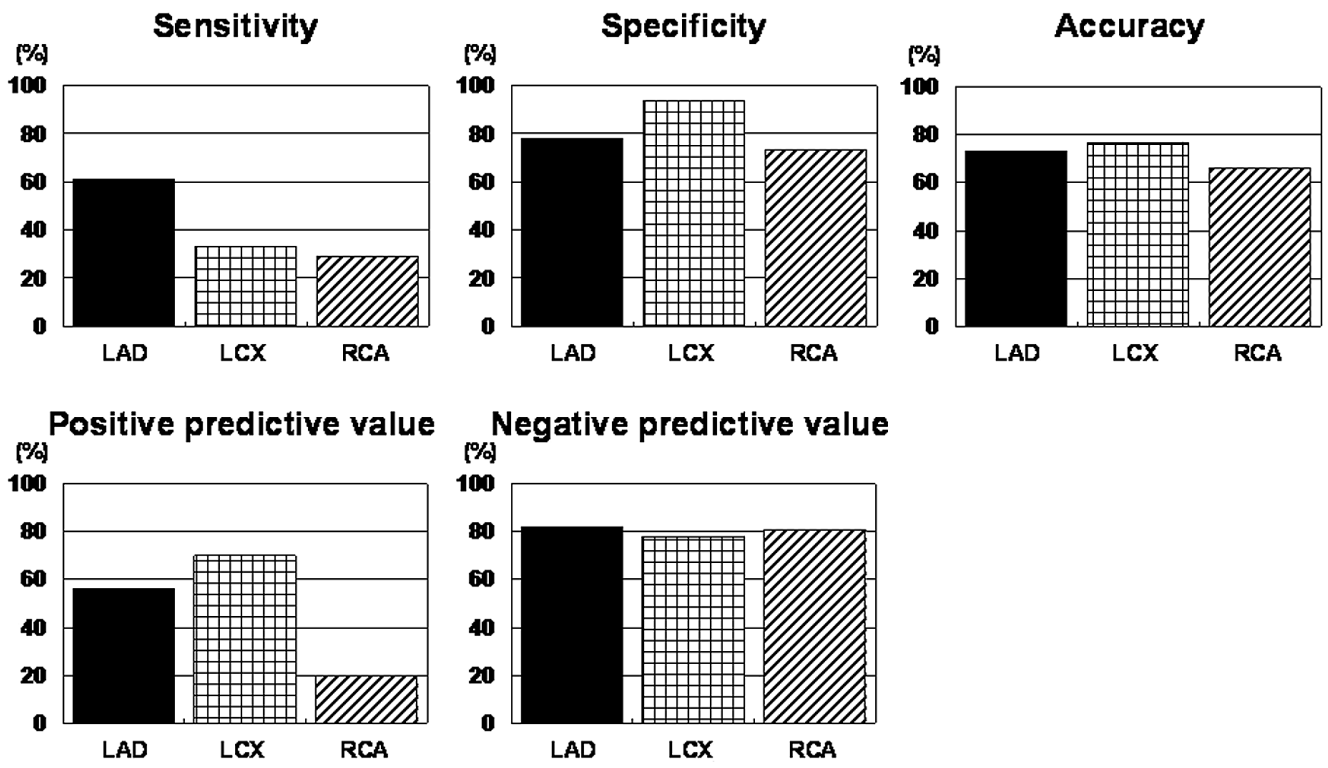

Fig 5. Sensitivity, specificity, predictive accuracy, positive and negative predictive values of detection of diastolic abnormality by dyssynchrony imaging for detecting stenoses of the left anterior descending artery (LAD), left circumflex artery (LCX) and right coronary artery (RCA).

of $30 \%$, and negative predictive value of $84 \%$ for the diagnosis of CAD. Receiver-operating characteristic analysis demonstrated the area under the curve was 0.61 for MOMI $(\mathrm{P}<0.05)$ and 0.68 for DADI $(\mathrm{P}<0.01)$. Intra- and interobserver agreements of DADI were $98 \%$ and $94 \%$, respectively.

\section{Detection of CAD by DADI per Major Coronary Artery Branch}

The diagnostic power of DADI for the 3 major coronary artery branches, LAD, LCX, and RCA, was assessed in a sub-analysis of 74 patients who underwent CAG (Fig 5). The sensitivity was the best for the LAD among the arteries, specificity ranged from $73 \%$ for the RCA to $94 \%$ for the LCX, predictive accuracy ranged from $65 \%$ for the RCA to $77 \%$ for the LCX, and negative predictive value was $82 \%$ for the LAD, $78 \%$ for the LCX, and $81 \%$ for the RCA.

\section{Discussion}

Using tissue Doppler-derived displacement timing analysis, we developed a novel modality that noninvasively displays PSS: detection of diastolic abnormality by dyssynchrony imaging (DADI). We also demonstrated in this prospective study that PSS detected by DADI reflects the presence of CAD in patients presenting with visually normal LV wall motion, whose chief complaint is chest pain. This technique does not require provocation, such as exercise or dobutamine.

The sensitivity of DADI for identifying patients with $\mathrm{CAD}$ was modest. It was the best in those who had lesions in the LAD, and was lower for the LCX and RCA. Nonetheless, specificity (range $73-94 \%$ ), predictive accuracy $(65-77 \%)$ and negative predictive value $(78-82 \%)$ all ranged around $70-80 \%$ regardless of the coronary artery branches (Fig 5). It is important to note that the sensitivity and specificity of DADI for detecting CAD were comparable to those of exercise electrocardiography, despite the nonprovocative nature of DADI.

PSS is a delayed ejection motion of the myocardium during the prolonged isovolumic relaxation time ${ }^{3-10}$ It is frequently observed in patients with CAD presenting with LV asynergy.3-25 Earlier studies have demonstrated the presence of abnormal diastolic function in patients with CAD without evidence of systolic wall motion abnormalities by radionuclide angiography or digitized cineangiography ${ }^{26-28}$ Kondo et al have also demonstrated that delayed outward LV wall motion in the isovolumic relaxation phase by digital subtraction high-frame-rate echocardiography was indicative of $\mathrm{CAD}^{29} \mathrm{In}$ our experience, a positive myocardial velocity occurring during the isovolumic relaxation phase as detected by the spectral tissue Doppler mapping technique (MOMI) was indicative of critical coronary artery stenosis in patients complaining of chest pain with visibly normal LV contraction!1 ${ }^{1}$ Furthermore, increased amplitude of PSS during dobutamine stress echocardiography detected the presence of CAD using tissue Doppler strain rate imaging 30 Those earlier observations are in accordance with our findings that a diastolic abnormality detected at rest by DADI has significant correlation with the presence of CAD in patients presenting with visibly normal LV wall motion.

On the other hand, PSS does occur in approximately one-third of the normal population? 31 Voigt et al reported that pathologic PSS and physiologic PSS were distinguished by using the cut-off value of $90 \mathrm{~ms}$ for the duration? ${ }^{3}$ Our study results demonstrated that the best cut-off value for the delay in the displacement peak was $100 \mathrm{~ms}$ for DADI, which was in good agreement with the cut-off value for abnormal PSS using strain rate reported by Voigt et al? ${ }^{3}$

In theory, displacement is inherently affected by tethering (ie, motion from the adjacent myocardium) 32 In fact, the base to the apex gradient in displacement is normally present. Using strain or stain rate appears to be more relevant for detecting localized abnormalities such as PSS than using displacement. Surprisingly, however, DADI detected the localization of PSS in the LV. In DADI, the delay of displacement peaks was measured, not from end-diastole, but from end-systole, for a limited duration as short as $100 \mathrm{~ms}$. Because the LV myocardium temporarily shows a 
velocity closest to zero at end-systole, DADI can highlight moving tissue from adjacent tissue that is not moving, thereby enabling location of PSS even though displacement is theoretically affected by tethering. Another robust aspect of using displacement rather than strain or strain rate is the reproducibility of the data. Displacement is directly calculated by the integration of Doppler velocity, resulting in much greater signal-to-noise ratio than strain or strain rate. Unlike strain or strain rate, the displacement calculation does not include subtraction or differentiation, both of which increase errors. In addition, neither the apex to base gradient nor the angle dependence of displacement affects the results in DADI, because timing analysis is independent from the amplitude of the data. Finally, Doppler measurement is superior to a 2-D speckle tracking technique in terms of time resolution. Thus, it is suitable for measuring the events lasting less than $100 \mathrm{~ms}$.

MOMI uses a spectral tissue Doppler technique, which can be performed with any commercially available ultrasound machines. On the other hand, MOMI involves a tedious mapping technique, and much fewer sampling points than DADI. DADI has enabled us to visualize the presence of PSS with a few clicks of buttons, and better reflected the presence of CAD than MOMI.

\section{Clinical Implications}

Because DADI can be performed without provocation, it is particularly safe and easy to perform in patients with coexisting morbidities, such as peripheral artery disease in the lower limb, orthopedic diseases, or cerebrovascular diseases in the elderly. This simple methodology has a comparable sensitivity and specificity to those of exercise electrocardiography, with a relatively high negative predictive value. Accordingly, it may be used as a noninvasive, nonprovocative diagnostic supplement in place of exercise electrocardiography in patients complaining of chest pain and presenting with normal echocardiograms, with an addition of a few more minutes to routine echocardiography. Future application of DADI to dobutamine or exercise echocardiography may further enhance the diagnostic power of DADI for the detection of CAD.

\section{Study Limitations}

First, this study was a single-center study including a relatively small number of patients. Second, $79 \%$ of the normal subjects (113 of 143 subjects) were diagnosed as normal by thallium-201 SPECT without CAG, so some may have had CAD. Nonetheless, normal findings in stress SPECT were associated with an average annual hard event rate of merely $0.6 \%$ (death or nonfatal myocardial infarction) $)^{33}$ In other words, CAD included in the normal subset of patients was considered minimal in view of clinical significance. In addition, the sub-analysis that included only those with CAG demonstrated a similar correlation. Third, PSS may also occur in nonischemic conditions such as LV hypertrophy, LV systolic dysfunction, and left bundle branch block ${ }^{34-36}$ We excluded those showing abnormal echocardiograms, so it is important to note that DADI should not be used for the detection of CAD in those showing abnormal echocardiograms. Nonetheless, this nonprovocative test would still be clinically relevant because many patients suspected of having angina pectoris for the first time demonstrate normal echocardiograms at rest.

In conclusion, we have developed a novel modality by using tissue Doppler derived displacement timing analysis: detection of diastolic abnormality by dyssynchrony imaging (DADI), which readily portrays the presence of PSS on 2-D echocardiograms. Detection of PSS by DADI showed a significant correlation with the presence of CAD among patients presenting with chest pain and visibly normal wall motion.

\section{Acknowledgments}

The authors are grateful for the excellent technical assistance provided by Ms Naoko Kobayashi, Ms Yayoi Ohno, Ms Sanae Ohata, Ms Sonoko Yamasaki, Ms Naomi Fujita, and Ms Tongsuk Son. We also thank Drs Hitoshi Minamiguchi, Hirokuni Akahori, Masamichi Yano, and Nobuaki Tanaka for referring patients and analyzing data. We also acknowledge the valuable technical advice from Mr Yasuhiko Abe, Mr Kenji Akao, and Mr Youhei Kusaka.

\section{References}

1. Gibbons RJ, Balady GJ, Beasley JW, Bricker JT, Duvernoy WF, Froelicher VF, et al. ACC/AHA guidelines for exercise testing: Executive summary. A report of the American College of Cardiology/American Heart Association Task Force on Practice Guidelines (Committee on Exercise Testing). Circulation 1997; 96: 345-354.

2. Picano E, Lattanzi F, Orlandini A, Marini C, L'Abbate A. Stress echocardiography and the human factor: The importance of being expert. J Am Coll Cardiol 1991; 17: 666-669.

3. Kukulski T, Jamal F, Herbots L, D'hooge J, Bijnens B, Hatle L, et al. Identification of acutely ischemic myocardium using ultrasonic strain measurements: A clinical study in patients undergoing coronary angioplasty. J Am Coll Cardiol 2003; 41: 810-819.

4. Song JK, Song JM, Kang DH, Haluska B, Marwick TH. Postsystolic thickening detected by Doppler myocardial imaging: A marker of viability or ischemia in patients with myocardial infarction. Clin Cardiol 2004; 27: 29-32.

5. Miwa K, Ishii K, Makita T, Okuda N. Effects of postischemic regional left ventricular diastolic wall motion abnormalities or delayed relaxation following coronary vasospasm on global diastolic function. Circ J 2005; 69: 439-445.

6. Tennant R, Wiggers CJ. The effect of coronary occlusion on myocardial contraction. Am J Physiol 1935; 112: 351-361.

7. Theroux P, Ross J Jr, Franklin D, Kemper WS, Sasyama S. Regional Myocardial function in the conscious dog during acute coronary occlusion and responses to morphine, propranolol, nitroglycerin, and lidocaine. Circulation 1976; 53: 302-314.

8. Forrester JS, Wyatt HL, da Luz PL, Tyberg JV, Diamond GA, Swan HJ. Functional significance of regional ischemic contraction abnormalities. Circulation 1976; 54: 64-70.

9. Takayama M, Norris RM, Brown MA, Armiger LC, Rivers JT, White HD. Postsystolic shortening of acutely ischemic canine myocardium predicts early and late recovery of function after coronary artery occlusion. Circulation 1988; 78: 994-1007.

10. Skulstad H, Edvardsen T, Urheim S, Rabben SI, Stugaard M, Lyseggen E, et al. Postsystolic shortening in ischemic myocardium: Active contraction or passive recoil? Circulation 2002; 106: 718-724.

11. Onishi T, Uematsu M, Nanto S, Iida O, Morozumi T, Kotani J, et al. Positive isovolumic relaxation velocity detected by a spectral tissue Doppler mapping technique as an indicator of coronary artery disease: A prospective study. J Am Soc Echocardiogr 2007; 20: 158-164.

12. Miyatake K, Yamagishi M, Tanaka N, Uematsu M, Yamazaki N, Mine $Y$, et al. New method for evaluating left ventricular wall motion by color-coded tissue Doppler imaging: In vitro and in vivo studies. $J$ Am Coll Cardiol 1995; 25: 717-724.

13. Sutherland GR, Stewart MJ, Groundstroem KW, Moran CM, Fleming A, Guell-Peris FJ, et al. Color Doppler myocardial imaging: A new technique for the assessment of myocardial function. $J$ Am Soc Echocardiogr 1994; 7: 441-458.

14. Uematsu M, Miyatake K, Tanaka N, Matsuda H, Sano A, Yamazaki $\mathrm{N}$, et al. Myocardial velocity gradient as a new indicator of regional left ventricular contraction: Detection by a two-dimensional tissue Doppler imaging technique. J Am Coll Cardiol 1995; 26: 217-223.

15. Gorcsan J 3rd, Strum DP, Mandarino WA, Gulati VK, Pinsky MR. Quantitative assessment of alterations in regional left ventricular contractility with color-coded tissue Doppler echocardiography: Comparison with sonomicrometry and pressure-volume relations. Circulation 1997; 95: 2423-2433.

16. Heimdal A, Stoylen A, Torp H, Skjaerpe T. Real-time strain rate imaging of the left ventricle by ultrasound. J Am Soc Echocardiogr 
1998; 11: $1013-1019$.

17. Urheim S, Edvardsen T, Torp H, Angelsen B, Smiseth OA. Myocardial strain by Doppler echocardiography: Validation of a new method to quantify regional myocardial function. Circulation 2000; 102: $1158-1164$.

18. Fujimoto S, Oki T, Tabata T, Tanaka H, Yamada H, Oishi Y, et al. Novel approach to the quantitation of regional left ventricular systolic and diastolic function using tissue Doppler imaging to create a myocardial velocity profile and gradient. Circ J 2003; 67: 416-422.

19. Sahn DJ, DeMaria A, Kisslo J, Weyman AE, the Committee on MMode Standardization of the American Society of Echocardiography. Recommendations regarding quantitation of M-mode echocardiography: Results of survey of echocardiographic measurements. Circulation 1978; 58: $1072-1083$.

20. Okuda K, Asanuma T, Hirano T, Masuda K, Otani K, Ishikura F, et al. Impact of the coronary flow reduction at rest on myocardial perfusion and functional indices derived from myocardial contrast and strain echocardiography. J Am Soc Echocardiogr 2006; 19: 781-787.

21. Sade LE, Severyn DA, Kanzaki H, Dohi K, Gorcsan J 3rd. Secondgeneration tissue Doppler with angle-corrected color-coded wall displacement for quantitative assessment of regional left ventricular function. Am J Cardiol 2003; 92: 554-560.

22. Garcia EV, Van Train K, Maddahi J, Prigent F, Friedman J, Areeda $\mathrm{J}$, et al. Quantification of rotational thallium-201 myocardial tomography. J Nucl Med 1985; 26: 17-26.

23. Derumeaux G, Ovize M, Loufoua J, Andre-Fouet X, Minaire Y, Cribier A, et al. Doppler tissue imaging quantitates regional wall motion during myocardial ischemia and reperfusion. Circulation 1998; 97: 1970-1977.

24. Edvardsen T, Urheim S, Skulstad H, Steine K, Ihlen H, Smiseth OA. Quantification of left ventricular systolic function by tissue Doppler echocardiography: Added value of measuring pre- and postejection velocities in ischemic myocardium. Circulation 2002; 105: 2071 2077.

25. Garcia-Fernandez MA, Azevedo J, Moreno M, Bermejo J, PerezCastellano N, Puerta P, et al. Regional diastolic function in ischaemic heart disease using pulsed wave Doppler tissue imaging. Eur Heart $J$ 1999; 20: 496-505.

26. Gibson DG, Brown DJ. Measurement of peak rates of left ventricular wall movement in man: Comparison of echocardiography with angiography. Br Heart J 1975; 37: 677-683.

27. Bonow RO, Bacharach SL, Green MV, Kent KM, Rosing DR,
Lipson LC, et al. Impaired left ventricular diastolic filling in patients with coronary artery disease: Assessment with radionuclide angiography. Circulation 1981; 64: 315-323.

28. Yamagishi T, Ozaki M, Kumada T, Ikezono T, Shimizu T, Furutani $\mathrm{Y}$, et al. Asynchronous left ventricular diastolic filling in patients with isolated disease of the left anterior descending coronary artery: Assessment with radionuclide ventriculography. Circulation 1984; 69: $933-942$.

29. Kondo H, Masuyama T, Ishihara K, Mano T, Yamamoto K, Naito J, et al. Digital subtraction high-frame-rate echocardiography in detecting delayed onset of regional left ventricular relaxation in ischemic heart disease. Circulation 1995; 91: 304-312.

30. Voigt JU, Exner B, Schmiedehausen K, Huchzermeyer C, Reulbach $\mathrm{U}$, Nixdorff U, et al. Strain-rate imaging during dobutamine stress echocardiography provides objective evidence of inducible ischemia. Circulation 2003; 107: 2120-2126.

31. Voigt JU, Lindenmeier G, Exner B, Regenfus M, Werner D, Reulbach $\mathrm{U}$, et al. Incidence and characteristics of segmental postsystolic longitudinal shortening in normal, acutely ischemic, and scarred myocardium. J Am Soc Echocardiogr 2003; 16: 415-423.

32. Skulstad H, Urheim S, Edvardsen T, Andersen K, Lyseggen E, Vartdal T, et al. Grading of myocardial dysfunction by tissue Doppler echocardiography: A comparison between velocity, displacement, and strain imaging in acute ischemia. J Am Coll Cardiol 2006; 47: $1672-1682$

33. Iskander $\mathrm{S}$, Iskandrian $\mathrm{AE}$. Risk assessment using single-photon emission computed tomographic technetium-99m sestamibi imaging. J Am Coll Cardiol 1998; 32: 57-62.

34. Citro R, Galderisi M, Guarini P, Cicala S, Mattioli D, Bianco A, et al. Left bundle branch block with and without coronary artery disease: Which value for a tissue Doppler-derived post-systolic motion? Ital Heart J 2003; 4: 706-712.

35. Pai RG, Gill KS. Amplitudes, durations, and timings of apically directed left ventricular myocardial velocities. II: Systolic and diastolic asynchrony in patients with left ventricular hypertrophy. $J \mathrm{Am}$ Soc Echocardiogr 1998; 11: 112-118.

36. Sade LE, Kanzaki H, Severyn D, Dohi K, Gorcsan J 3rd. Quantification of radial mechanical dyssynchrony in patients with left bundle branch block and idiopathic dilated cardiomyopathy without conduction delay by tissue displacement imaging. Am J Cardiol 2004; 94: 514-518. 